Sheridan College

SOURCE: Sheridan Institutional Repository

$1-1-2010$

\title{
Computational Public Safety in Emergency Management Communications
}

Cristina Ribeiro

Sheridan College, cristina.ribeiro@sheridancollege.ca

Alexander Ferworn

Ryerson University

Follow this and additional works at: https://source.sheridancollege.ca/fast_publications

Part of the Computer and Systems Architecture Commons, Digital Communications and Networking Commons, and the Technology and Innovation Commons

\section{SOURCE Citation}

Ribeiro, Cristina and Ferworn, Alexander, "Computational Public Safety in Emergency Management Communications" (2010). Publications and Scholarship. 55.

https://source.sheridancollege.ca/fast_publications/55

\section{(c) (1) $9 \odot$}

This work is licensed under a Creative Commons Attribution-Noncommercial-No Derivative Works 4.0 License. This Conference Paper is brought to you for free and open access by the Faculty of Applied Science \& Technology (FAST) at SOURCE: Sheridan Institutional Repository. It has been accepted for inclusion in Publications and Scholarship by an authorized administrator of SOURCE: Sheridan Institutional Repository. For more information, please contact source@sheridancollege.ca. 


\section{Computational Public Safety in Emergency Management Communications}

\author{
Cristina Ribeiro \\ Computer Science \\ University of Waterloo \\ Waterloo, Canada \\ cribeiro@uwaterloo.ca
}

\author{
Alexander Ferworn \\ Computer Science \\ Ryerson University \\ Toronto, Canada \\ aferworn@ryerson.ca
}

\begin{abstract}
Communications are very important in any situation but in emergency management it is imperative that the communications be reliable and responsive to the evolving situation. In emergency management there are many different types of networks with different objectives. It is of immense value to have the ability to seamlessly integrate other networks and computing resources into one interconnected heterogeneous network. The entire management team should be able to access any of the individual networks and their resources. In this paper we discuss various wireless network communication options in the context of their viability for use in emergency management. We analyze various technical aspects such as propagation delay, packet delivery ratio, and transmission rates. In addition the environmental conditions that impair communications are discussed. All experiments we conducted took place in a setting that was real, using real equipment that was physically situated in settings that can be expected in urban disaster settings-our results are not simulations. They were performed in cooperation with the Ontario Provincial Police, Provincial Emergency Response Team.
\end{abstract}

Keywords-Wireless Mesh Netwroks; Wi-Fi; Buetooth; Emergency Management Computational Public Safety; Urban Search and Rescue, Canine Pose Estimation.

\section{INTRODUCTION}

Computational Public Safety (CPS) is crucial to the ongoing organization of emergency management services, and its copious amounts of data. CPS is used in many areas of emergency management. Our research has focused on how it is specifically used in Urban Search and Rescue (US\&R), which shares the same objectives and issues as all other emergency management communications, yet possesses its own unique set of challenges that need to be addressed. Wireless networks using the TCP protocol are common and encounter many challenges, the propagation delay, packet delivery ratio, and transmission rates are characteristics that are affected. The experiments conducted in our research, quantifies the effects of different conditions on each of characteristics.

Wireless communication needs to be reliable in the environment intended for its use. It must have a $\mathrm{g}$ transmission range that is able to cope with the many challenges posed by the realities of smashed concrete and rebar and be robust enough to accommodate the demands of different emergency management applications. Usually this requires transmission over distances greater than $500 \mathrm{~m}$ [1]. The setup of the communications network must be simple and speedy involving known physical cache sizes so that the US\&R teams can focus their time on searching for and rescuing victims rather than hauling and manipulating finicky radio gear. In the midst of a disaster is not the place debug a network. Each level of added complexity must be justified in terms of the benefits it provides. These benefits must outweigh the inevitable problems that will be introduced. The complete network topology should be simple and reliable for US\&R workers to use rather than asking them to become experts on the spot to resolve communication problems that should not exist. In this paper we focus on the tactical communication required within an urban disaster between elements of a search team and emergency managers.

\section{BACKGROUND}

\section{A. Urban Search and Rescue}

The fastest and most reliable means of finding people trapped after a building collapse is through the use of trained Urban Search and Rescue (US\&R) dogs. These canines are the state-of-the-art when conducting search operations within an urban disaster. Search operations necessarily occur before rescue can take place. Since there is a finite time that someone can survive entombed within the wreckage of a building, it is critical that search operations occur as quickly and efficiently as possible to ensure that there is a high probability that rescue operations will be successful. Search operations have several challenges that increase the time it takes to find survivors within the wreckage and delay transmission of critical information to managers.

A particular matter requiring improvement is the situational awareness [2] of emergency managers while searches are being conducted. Situations can arise where even a human handler is unaware of their dog's whereabouts or behaviour because the dog is out of sight and the handler will have nothing to report - even if the dog finds something. This lack of situational awareness is generally due to the distance and obstacles between the handler and the dog. In the extreme, a handler may be asked to send his or her dog into the rubble of a building without the ability to actually follow behind, because human access may be extremely limited. If the handler cannot follow, there is no chance that emergency managers will know what is going on at the point of actual search. If the handler's situational awareness of the canine could be enhanced, search times could be reduced, improving the performance of the team, communicating information back to managers faster and the result might be more lives saved.

A complementary area of research is the augmentation of US\&R dogs $[1,3-5]$ with technology that allows emergency first responders to experience what is happening around the dog while it is searching. This area of research is very important as it allows searchers to become aware of what is actually happening around the dog without interfering with the search. This technology provides some additional situational awareness capabilities. The dog itself is equipped with 
many integrated sensing technologies, which transmit different data types and streams via a wireless mesh network. Each search dog being used on the site is equipped with such a system. These systems provide new challenges for disaster networks as their communication load exponentially increases the amount of data being transmitted and processed in the vicinity of the search site - a site which probably has very limited or no communication infrastructure.

Computational Public Safety (CPS) involves the application of computational resources, theory and practice in support of and improvement to public safety processes. The objective of this work was to develop an understanding of the ability of a disaster network to deal with at least some of the expected data streams that will become part of the response to an urban disaster. In this paper we focus on a subset of the communication expected from canine sensors in aide of improving the situational awareness of search mangers. We focus on a single data streams associate with the determination of canine pose.

The work can improve how US\&R is conducted by utilizing technology to provide situational awareness to US\&R canine handlers, supporting emergency first responders and search managers as the pose or position the dog is in can be used to determine what the dog is actually doing while searching and gives an indication of the progress the dog is making through environments typically including rubble with loose debris and many voids - potentially containing people in various states. Many search dogs are cross-trained to indicated both live and dead humans in different ways. The ability to detect canine pose offers the hope that what the dog is trying to say can be directly communicated to people who can decide what to do next.

A number of challenges exist in determining canine pose and communicating the relevant information back to the handler. These challenges include choosing the appropriate wireless networking communications and evaluating the network's competency to transmit data in a timely manner to all essential parties. The Canine Pose Estimation (CPE) system transmits body position data over a wireless mesh network (WMN) to a computer, where the dog's pose is actually determined algorithmically.

\section{B. Emergency Management Communications}

As there is usually no wired infrastructure in place at an urban disaster, most network communication occurs wirelessly through radio transmission. Wireless networks experience many challenges that are not present with wired networks. Adverse environmental conditions add to the challenges that these networks face; challenges include weather, temperature, humidity, surrounding debris, consisting of materials known to cause interference ( steel in various forms including rebar, and concrete) [6]. Network interference also includes the increasing number of wireless enabled devices like cell phones, desktops, laptops, smart phones, and response robots, most enabled with significant network and application capacity including Bluetooth, GPS, Wi-Fi and access to cellular networks base stations [6]. Wireless networks are complicated, usually difficult to configure and are effected by all manner of environmental issues. This is why, increasingly, WMNs are a popular suggested solution to these problems.

Having said this, to the best of our knowledge, the majority of published research work in the area of measuring the performance of WMNs is based on simulations involving synthetic traffic and the placement of nodes within a lab or the outright simulation of the actual radio. In our work we concentrate on actual propagation delay in an actual system.

Microsoft Corporation conducted a study [7], where propagation delay was evaluated across a WMN, which was deployed in an office building and used real user network traffic. This research concluded that the captured user traffic was substantially different than the synthetic traffic used in similar experiments conducted through simulations. The results showed an additional median propagation delay of $20 \mathrm{~ms}$ with each transmission across the WMN, compared to simulation results. $20 \mathrm{~ms}$ seems like a short amount of delay but with many applications coming on line, this bottleneck is only exposed when real equipment was used.

Our experiments deployed an actual WMN in a building selected because its structural components closely resembled that of a partially collapsed structures that we have seen. The results achieved produced propagation delays expected for the different configurations tested and the scenarios they represent in a disaster environment. In the next sections we discuss the technology we selected to test within the context of our CPE system.

\section{EMERGENCY MANAGEMENT COMMUNiCATION TeCHNOLOGIES}

There are many options available for the transmission of sensor data. They include Bluetooth, Xbee, radio modems, WiMax, and WiFi. In this section we look at the benefits and detriments of each and discuss their viability.

\section{A. Xbee}

Xbee is a specification for a suite of high level communication protocols using small, low-power digital radios based on the IEEE 802.15.4-2003 standard for wireless personal area networks (WPANs), such as wireless headphones connecting with cell phones via shortrange radio [6]. The technology defined by the XBee specification is intended to be simpler and less expensive than other WPANs, such as Bluetooth. XBee is targeted at radio-frequency (RF) applications that require a low data rate, long battery life, and secure networking. This would be a viable option for a simple embedded system, which involves a large part of emergency management applications but not mobile applications with high data rates.

\section{B. Bluetooth}

Bluetooth transmission range is short, this makes it undesirable for use in US\&R mobile applications, as dogs move about ahead of their handlers, they quickly go out of range of receivers. Effective communications between Bluetooth devices is limited to approximately $10 \mathrm{~m}$ [6], too short a distance for a roaming dog.

\section{WiMax}

WiMax is based on the IEEE 802.16 standard. It provides different types of access, from portable (similar to a cordless phone) to fixed (an alternative to wired access, where the end user's wireless termination point is fixed in location.) It is only intended for point-topoint applications and therefore is not applicable to applications like CPE. However, the range is long--3km [6], making WiMax a contender for other, less mobile, applications.

\section{D. $W i-F i$}

Wireless Fidelity (Wi-Fi) technology has higher throughput and greater signal strength than the other short-range network technologies. Greater signal strength is important in a disaster area where debris from collapsed structures can interfere and affect the connectivity of any device. During training deployment exercises in Toronto, the search dogs could reach distances of $250 \mathrm{~m}$ ahead of their handlers [1]. The Wi-Fi range can be extended indefinitely by deploying more Wi-Fi nodes in the area using existing meshing technology [6].

\section{WIRELESS FIDELITY ISSUES}

Wireless fidelity issues arise from the TCP protocol, which is widely used and is effective in transmitting data packets to their 
intended destinations. When TCP is utilized over a wireless network it exhibits some performance degradation. One factor effecting performance pertains to the propagation delay across the network, which may be increased. Another performance issue relates to packet delivery ratios, which may decrease. This occurs as packets are lost in the transmission of data across the network. In [8], a survey of different TCP performance improvement schemes for wireless networks is presented and goes on to indicate that wireless networks are inherently less reliable than those that are wired resulting in more packet loss.

TCP assumes that any packet loss that occurs is the result of congestion. TCP handles congestion by invoking congestion control. This works well in wired networks, but in wireless networks this results in decreased performance. Decreased performance occurs due to the characteristics of wireless networks, where packets are lost as a result of random high bit error rates and intermittent connectivity, which is due to the mobility of nodes and the nature of radio communications in a an urban disaster. This congestion control could introduce long periods of disconnection. The result of this is an increase in propagation delays, and decreased packet delivery ratios and transmission rates. Essentially all communication is slowed on the network.

\section{A. Experimental Setup}

Experiments were conducted by deploying an actual WMN. Selecting an appropriate location for the deployment was essential in order to mimic that of an environment that would be found in an urban disaster involving the structural collapse of commercial buildings adhering to North American construction standards. Clearly, this is very specific to a geographic area. North American buildings tend to have far more reinforcement-commonly reinforcing steel rods and the concrete is machine-mixed and uniform.

The venue chosen was the Centre for Computing and Engineering (CCE, Ryerson University, Toronto) because the building was constructed predominantly of exposed concrete pillars and walls with intersecting hallways that were accessible at sharp angles-we believe, mimicking slabs of concrete that tend to characterize the collapse of this type of building [9]. The building's structure was advantageous as all concrete walls and pillars were easily identifiable and could be used as barriers to simulate the environment found in a partial collapse of a similar building. The CPE device transmitted canine pose data across the WMN deployed in the building in real time.

Three WMN configurations were deployed in the building, described in the experimental results. We ensured that each mesh router connected to the next mesh router, in order, to ensure data would be transmitted across the network in correspondence with each configuration. The connection signal strength between each of the mesh routers was confirmed as a good network connection with a signal to noise ratio (SNR) below $60 \mathrm{~dB}$ and with signal strength no less than $70 \mathrm{~dB}$.

The last mesh router in the network acted like a gateway that connected the mesh network to the Internet. This mesh router was connected wirelessly to the Ryerson Network-Centric Applied Research Team (N-CART) lab's wireless network. The laptop connected to the Ryerson University wireless network. By setting up the network in this fashion, using two different network connections to the Internet, we ensured that the data received on the client end has successfully been transmitted from its destination point.

We ran two tests per configuration as seen in figures $\mathrm{X}-\mathrm{Y}$. We compare the repeated tests and discuss the reliability of the results. The mean PD and PDR were calculated for a data set, where a data set was comprised of ten canine pose data strings that were transmitted by the CPE device.
All of the materials and environmental conditions found at a disaster site can significantly affect the propagation delay of a WMN. It is not only the materials themselves that affect propagation delay, but their dimensions and placement play a part--each a factor increasing the propagation delay within a network [10]. Some materials refract wireless signals, while others prevent them from penetrating. Moreover, there is an inverse relationship between the number of "hops" a packet must take and network performance when it comes to propagation delay $[11,12]$. Thus, network performance is adversely affected again.

\section{B. Propagation Delay}

Propagation delay is the time taken to transmit a packet between source and destination nodes in a network [6]. To determine the propagation delay we measure the time it takes to send data from the source to its destination (Tx), and also measure the time it takes to receive the data sent back from the destination to the source $(\mathrm{Rx})$. The difference between $\mathrm{Rx}$ and $\mathrm{Tx}$ gives us the propagation delay of the data traveling across the network twice. To determine the experimental propagation delay from source to destination only, the resulting value is divided by two.

$$
(\mathrm{Rx}-\mathrm{Tx}) / 2=\mathrm{PD} .
$$

The first configuration was a simple linear formation free from any obstacles as shown in Figure 1. This configuration represented the base case under optimal environmental conditions. The other configurations were compared to this first configuration in terms of propagation delay and PDR. The extended network coverage possible under such environmental conditions while maintaining good signal strength between each of the mesh nodes was also assessed.

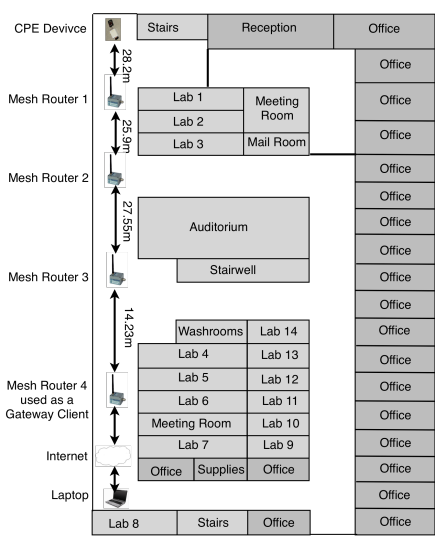

Figure 1. Configuration 1

In our first test had a mean propagation delay of $170.24 \mathrm{~ms}$. The second test produced a mean of $318.42 \mathrm{~ms}$. When comparing them with each other, there was a difference of $148.18 \mathrm{~ms}$ between the two means. Figure 2 shows the mean propagation delay for each data set. The mean propagation delay experienced by the WMN in configuration 1, was $244.33 \mathrm{~ms}$.

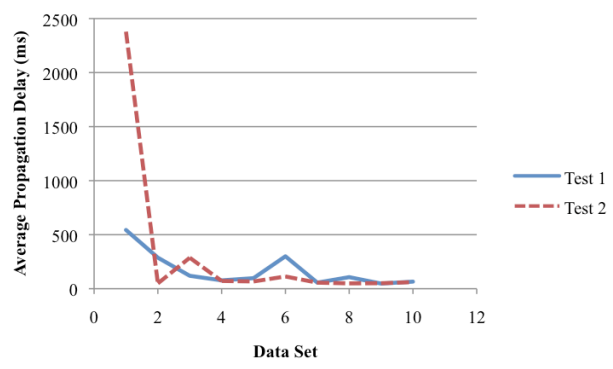

Figure 2. Propapgation Delay for Configuration 1 
The second WMN configuration shown in Figure 3, depicts extended network coverage. The mesh routers were deployed in a manner to extend network connectivity around obstacles that do not allow wireless radio signals to penetrate through. This configuration represents large thick obstacles made of reinforced concrete impeding wireless transmission and also where some rooms would be inaccessible and the USAR dogs would have to go around obstacles in order to continue searching.

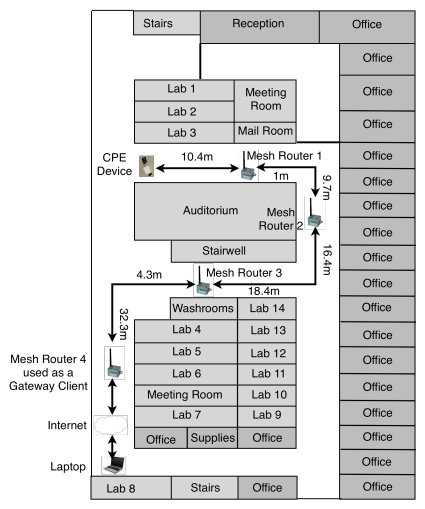

Figure 3. Configuration 2

In the first test, the mean propagation delay is $456.4 \mathrm{~ms}$ as shown in Figure 4. The second test has a mean propagation delay of 515.23 $\mathrm{ms}$. This results in a difference of $58.83 \mathrm{~ms}$ between the configuration 2 results. The WMN has a mean propagation delay of $485.58 \mathrm{~ms}$. There is a difference of $241.49 \mathrm{~ms}$ between this mean propagation delay and configuration 1 . This is a distinct measureable difference, which shows that the propagation delay significantly increased in this situation.

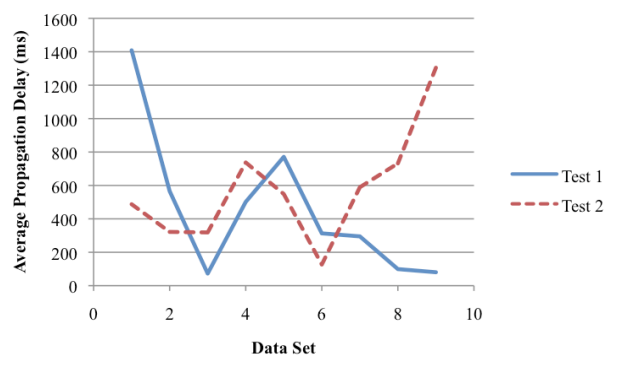

Figure 4. Propapgation Delay for Configuration 2



Figure 5. Configuration 3

The third configuration is shown in Figure 5 in where an attempt was made to penetrate through some obstacles by deploying a mesh router in one of the labs. In cases where a room has not caved in we may wish to extend the network into this room so that if a dog is searching in a large room the handler will still be able to receive the pose data at the other end of the network. Node placement is important to ensure that each of the nodes in the network is connected.

For configuration 3 the first test has a mean propagation delay of $748.69 \mathrm{~ms}$, shown in Figure 6. The second test has a mean of 664.5 $\mathrm{ms}$. The difference between the two tests is $84.19 \mathrm{~ms}$. In this configuration the WMN experienced an mean propagation time of $706.6 \mathrm{~ms}$. A difference of $221.02 \mathrm{~ms}$ compared to configuration 2 and a difference of $462.51 \mathrm{~ms}$ compared to configuration 1 .

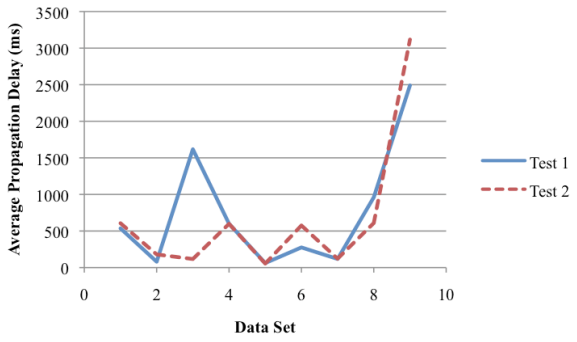

Figure 6. Propapgation Delay for Configuration 3

\section{Packet Delivery Ratio}

Using TCP protocols to transmit the data across the WMN could cause packet loss due to the window size, which may become congested and full. When this occurs the PDR decreases as packets are lost [7-8]. There are three indications of packet loss when using TCP. The first indication is a retransmission timeout (RTO) at the source. The second is the arrival of duplicate acknowledgements (ACKs) at the source. Finally, the third indication is the receipt of the Internet Control Message Protocol (ICMP) source quench message [8].

TCP measures the length of time for an ACK to return from the destination also known as the Round Trip Time (RTT). The protocol keeps track of the average of this delay and estimates the deviation of the delay based on these averages. This delay is then used to determine if congestion is likely to occur. The protocol deems it likely there is congestion when the RTT delay is greater than four times the deviation estimated. In this case TCP runs congestion avoidance, which increases the congestion window $[13,14]$. This is done to ensure that packets are not lost and that the PDR remains high.

All of the tests in these experiments requested 100 data strings of the canine pose data. The PDR algorithm found a PDR of $100 \%$ for both tests for configuration 1 as seen in Figure 7. In configuration 2 there is a PDR of $88 \%$, for both tests. While configuration 3 has a PDR of $84 \%$ for test 1 and $83 \%$ for test 2 .

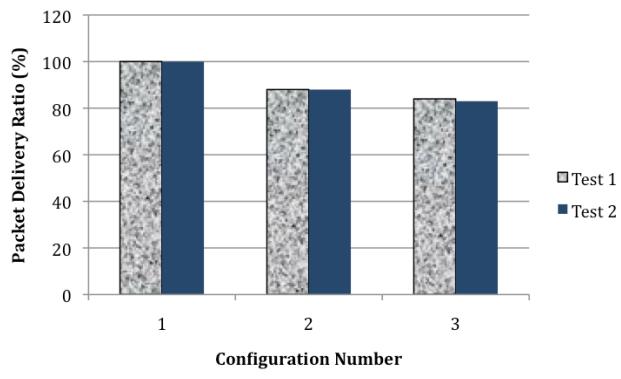

Figure 7. Packet Delivery Ratio for Configurations

This shows that configuration 1 , which experienced the least interference, is also the most reliable as it received all 100 packets at the other end of the network. Configuration 2 was not as quite as successful receiving only $88 \%$ of its packets. This configuration experienced a higher degree of interference due to the node placement. Finally, configuration 3 has the lowest PDR of the configurations. It 
faced the most challenging environmental conditions with many obstacles directly between the nodes. There is a direct relationship between a high signal strength and PDR. The greater the signal strength, the greater was the PDR as a result (and vice-versa).

Comparison of the results found between each of the configurations provides insight into the importance of node placement as well as the impact obstacles have on the network.

\section{MeSh NeTWORKS}

A wireless mesh network (WMN) is a self-healing, selfconfiguring, self-regulating and adaptive network [15]. WMNs can function without human intervention or administration and can be easily deployed in an urban disaster with nodes placed where required to extend the network; typically, this would be done in a number of pragmatic ways including first responders dropping nodes off around the disaster zone to facilitate interconnection. This property of WMNs is a major asset for disaster environments, as each disaster is unique in terms of its layout, materials and dimensions. Once the mesh routers have been deployed they connect together to form a network through self-configuration.

If there are any changes in the network such as the loss of connectivity (such as a battery failure) between any of the other nodes, they adapt and heal the configuration and remain connected. The WMN has dedicated configuration and routing nodes.

Another advantage to deploying a $\mathrm{WMN}$ is that it can easily be integrated with grid computing and heterogeneous networking. Any other Wi-Fi enabled emergency management devices would seamlessly connect to the WMN. This is very beneficial to the planning and expanding of emergency management communications. It enables them to implement and provide new services and increase their efficiency and effectiveness.

\section{A. Transmission Range \& Connectivity}

The performance of the network coverage was also assessed for each of the configurations using the signal strength and signal to noise ratio (SNR) metrics. Signal strength is counter intuitive, the higher the value the lower the performance. The lower the signal strength value the better was the established connection. A value higher than $70 \mathrm{~dB}$ was considered a poor connection and the network would drop intermittently or not connect at all. For the SNR any value below 60 $\mathrm{dB}$ made for a good connection between network nodes, representing low noise in the network.

Data was transmitted utilizing a WMN, which broadcasted the data. The data was transmitted over the mesh network hopping from one mesh router to another until it reached its destination (the laptop). This multi-hop data transmission can experience signal loss and/or delays. It was important to evaluate and analyze whether the delay was significant enough to affect urban search and rescue. In the presence of obstacles and debris, the signal strength deteriorates from interference from many sources.

Looking at the direct distance between the nodes for this configuration to get a better idea of network coverage. When looking at the direct distance between each of the nodes, the network covers a distance of $88.34 \mathrm{~m}$. This is much lower than that of the linear distance and indicates that barriers cause significant interference to the WMN. This interference, weakens the signal and diminishes the distance the signal can travel, thus the network coverage is decreased.

This was verified by the network performance metrics used to determine node placement. Signal strength was measured to be $67 \mathrm{~dB}$; this was close to the tolerable threshold for a good connection, which was required to be less than $70 \mathrm{~dB}$. The difference when compared to configuration 1 and configuration 2 was $27 \mathrm{~dB}$ and $12 \mathrm{~dB}$ respectively.
The SNR was measured to be $56 \mathrm{~dB}$, which is also near the tolerable threshold for a good connection $(60 \mathrm{~dB})$. A difference of configuration 3 compared to 1 and 2 was $36 \mathrm{~dB}$ and $14 \mathrm{~dB}$ respectively.

Configuration 3 had the highest levels of interference in the network, compared to the other scenarios. This was due to the nodes being placed in a room, or between rooms and having nodes connect to each other through the walls of varying materials. The network coverage of the first node with the second was a very short distance of $2.3 \mathrm{~m}$ apart and $3.42 \mathrm{~m}$ across; as compared to the distance between node 2 and 3 or 3 and 4 that were much greater. This was due to the difference in the thickness of the concrete walls.

\section{CONCLUSIONS}

$\mathrm{Wi}-\mathrm{Fi}$ is the best option for emergency management communications, as it is the most cost effective and its transmission rate and range are superior to all the other options. There are still issues that need to be addressed and improved, such as wireless TCP, which leads to increases in PD and decreases in PDR. In addition increasing the transmission rates and range would significantly decrease the costs associated with deploying a WMN, as the number of nodes required would significantly decrease.

Future work includes analyzing and measuring these wireless network qualities with multiple technologies being run simultaneously. This would mimic the actual use of the system by the US\&R team. It would help delineate any new impediments which if improved would significantly assist emergency workers in their task of saving lives.

\section{REFERENCES}

[1] A. Ferworn, D. Ostrom, A. Sadeghian et al., "Rubble Search with Canine Augmentation Technology" IEEE International Conference on System of Systems Engineering, San Antonio, USA. 2007, pp. 1-6.

[2] M. R. Endsley, and DJ. Garland, "Theoretical underpinnings of situation awareness: A critical review", Situation Awareness Analysis and Measurement, Lawrence Erlbaum Associates, pp. 332, 2000.

[3] A. Ferworn, D. Ostrom, K. Barnum et al., "Canine Remote Deployment System for Urban Search and Rescue", Journal of Homeland Security and Emergency Management. Volume 5, Article 9, 2008.

[4] A. Ferworn, D. Ostrom, A. Sadeghian et al., "Canine as Robot in Directed Search", IEEE International Conference on System of Systems Engineering, San Antonio, USA, 2007, pp. 1-5.

[5] A. Ferworn, A. Sadeghian, K. Barnum et al., "Urban Search and Rescue with Canine Augmentation Technology", IEEE System of Systems Engineering (SoSE06), Los Angeles, USA, 2006.

[6] M. Ma, M. K. Denko, and Y. Zhang, "Wireless Quality of Service: Techniques, Standards and Applications", Auerbach Publications, Taylor\&Francis Group, USA, 2008.

[7] J. Eriksson, S. Agarwal, P. Bahl et al., "Feasibility study of mesh networks for allwireless offices", ACM/Usenix MobiSys, 2006, pp. 69-82.

[8] M. Patel, N. Tanna, P. Patel et al., "TCP over Wireless Networks: Issues, Challenges and Survey of Solutions", Technical report, Computer Science Department, University of Texas, Dallas, pp. 1-22, 2001.

[9] U.S. Army Corps of Engineers, Urban Search and Rescue Structures Specialist Field Operations Guide, September 2003 (US\&R-2-FG)

[10] A. Ferworn, N. Tran, J. Tran et al., "WiFi repeater deployment for improved communication in confined-space urban disaster search", IEEE System of Systems Engineering (SoSE07), 2007, pp. 1-5.

[11] D. Couto, D. Aguayo, J. Bicket et al., "A high-throughput path metric for multihop wireless routing", Wireless Networks, vol. 11, no. 4, pp. 419-434, 2005.

[12] J. Bicket, D. Aguayo, S. Biswas et al., "Architecture and evaluation of an unplanned 802.11 b mesh network", ACM New York, NY, USA, 2005, pp. 31-42.

[13] J. Postel, "Transmission Control Protocol", RFC 791, 1981.

[14] G. R. Wright, and W. R. Stevens, "TCP/IP illustrated. Vol. 2: The implementation", Addison-Wesley Professional Computing Series, Reading, Mass.: AddisonWesley, 1995.

[15] I. F. Akyildiz, X. Wang, and W. Wang, "Wireless mesh networks: a survey”, Computer Networks, vol. 47, no. 4, pp. 445-487, 2005. 